\title{
Gastrocolic fistula, a rare complication of repeated gastric surgery
}

\author{
Ratha Mun, Xu Wang, and Wei-Feng Huang
}

Department of Gastroenterology, The First Affiliated Hospital of Xiamen University, School of Medicine, Xiamen University, Xiamen, China
Received : November 13, 2021

Revised : December 10, 2021

Accepted: December 13, 2021

Correspondence to

Wei-Feng Huang, Ph.D.

Tel: +86-18150906129

Fax: +86-5922137748

E-mail: hwf0625@163.com

https://orcid.org/0000-0002-97557299
A 54-year-old man with weight loss and fatigue presented to the First Affiliated Hospital because of chronic watery diarrhea for 1 year, characterized by stool frequency of 6 to 10 times per day and postprandial diarrhea with undigested food particles. He had medical history of two times Billroth II gastrectomy due to gastric ulcer with perforation 18 and 10 years earlier respectively, as well as cholecystectomy due to gallstone 8 years ago. He had presented few months earlier with the same problem and no significant abnormalities were disclosed. On physical examinations he was pale and malnourished with normal vital signs. The abdomen was soft with two lines surgical scars. Laboratory values on admission showed anemia (10.0 $\mathrm{g} / \mathrm{dL})$, hypoalbuminemia $(2.79 \mathrm{~g} / \mathrm{dL})$, as well as negative fecal occult-blood test and normal serum gastrin. On colonoscopy, a $1.2 \times 1.0 \mathrm{~cm}$ fistula tract was discovered accidentally on transverse colon (Fig. 1A). This could be traversed endoscopically to enter the gastric lumen (Fig. 1B, arrow). Subsequently, computed tomography scan (Fig. 1C, arrow) and gastrografin meal (Fig. 1D) was performed to confirm the presence of a gastrocolic fistula. He was started on proton pump inhibitor (PPI) and total parenteral nutrition (TPN), then endoscopic closure of fistula using over-the scope-clip (OTSC) system was undergone, but the fistula reappeared after 6 weeks follow-up. Gastrocolic fistula is an abnormal communication between stomach and the colon. The treatment should be individualized. Surgery can be the mainstay of treatment in most cases. After correction of malnutrition the patient was referred to the surgery. Subtotal gastrectomy with gastrojejunal anastomose resection, subtotal resection of transverse colon and enterolysis were successfully underdone. Histopathology examination had excluded malignancy. After the surgery, antibiotics, high-dose PPI, as well as TPN were administered. The patient was discharged home after 21 days of hospitalization. Hydrotalcite chewable tablets with oral enteral nutritional powder were continued. During 1 year of follow-up, the patient had no complain of watery diarrhea and had about $5 \mathrm{~kg}$ weight gain.

The authors are accountable for all aspects of the work in ensuring that questions related to the accuracy or integrity of any part of the work are appropriately investigated and resolved. Written informed consent was obtained from the patient for publication of this "Images of Interest."

\section{Conflict of interest}

No potential conflict of interest relevant to this article was reported. 

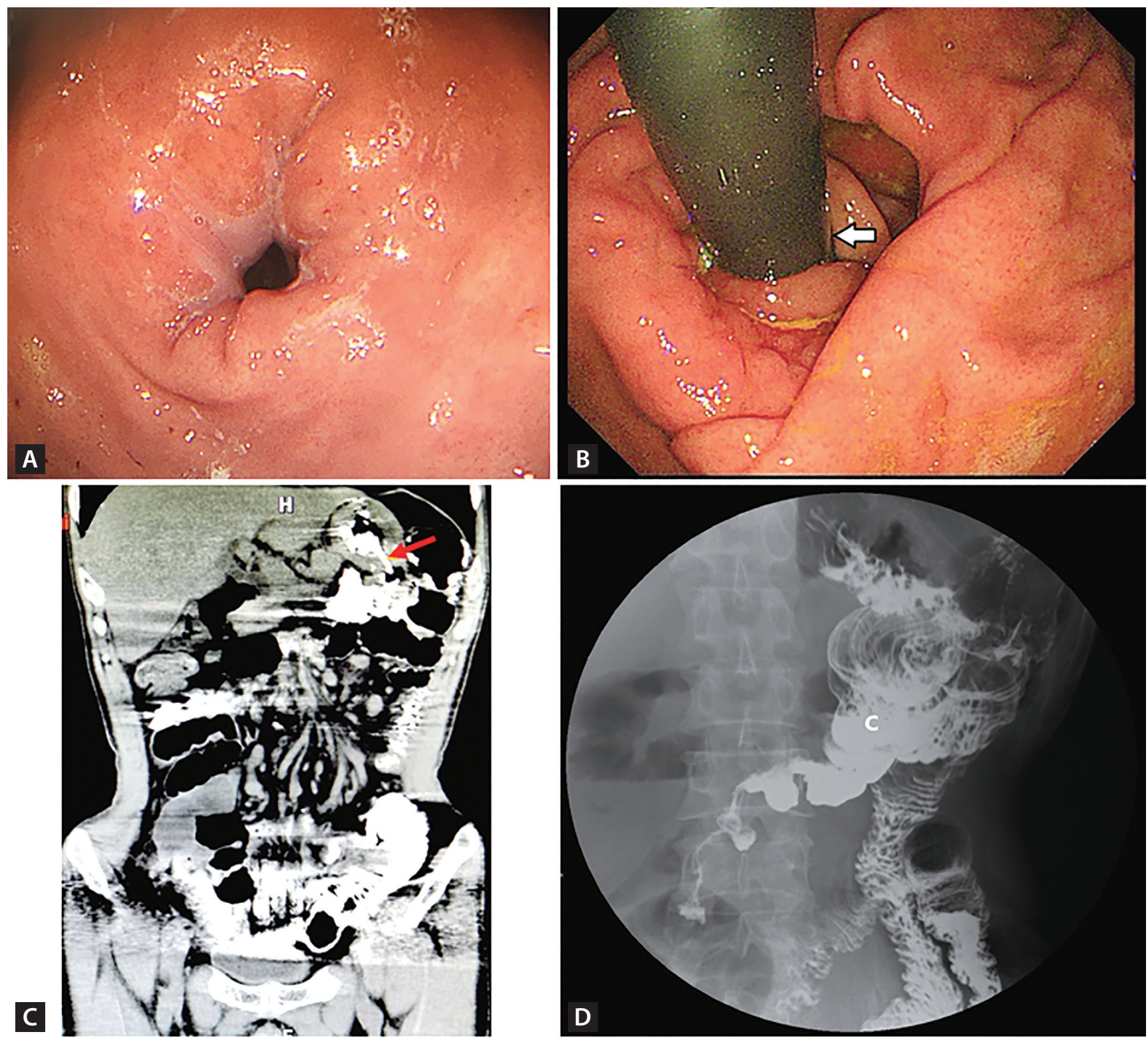

Figure 1. Gastrocolic fistula, a rare complication of repeated gastric surgery. (A) Colonoscopy showed the fistula tract from the transverse colon side. (B) The scope of colonoscopy passed through the fistula tract, showed the gastric lumen (arrow). (C, D) Computed tomography scan (arrow) and gastrografin meal showed the rapid flow of contrast from the stomach to the transverse colon.

\section{Acknowledgments}

This work was supported by the Medical and Health Guid-

ing Project of Xiamen (3502Z20214ZD1028). 